\title{
Properties of Mitochondrial-Derived Peptides (Mdps), Type 2 Diabetes, and Relationship with Oxidative Stress
}

\author{
Luc Rochette $^{1 *}$, Alexandre Meloux ${ }^{1}$, Eve Rigal ${ }^{1}$, Marianne Zeller ${ }^{1}$, Yves Cottin ${ }^{1,2}$ and Catherine Vergely ${ }^{1}$ \\ ${ }^{1}$ Equipe d'Accueil (EA 7460): Physiopathologie et Epidémiologie Cérébro-Cardiovasculaires (PEC2), Université de Bourgogne - Franche \\ Comté, Faculté des Sciences de Santé, 7 Bd Jeanne d'Arc, 21000 Dijon, France
}

${ }^{2}$ Service de Cardiologie, CHU-Dijon, 21000 France

*Corresponding author: Luc Rochette, Equipe d'Accueil (EA 7460): Physiopathologie et Epidémiologie Cérébro-Cardiovasculaires (PEC2), Université de Bourgogne - Franche Comté, Faculté des Sciences de Santé, 7 Bd Jeanne d’Arc, 21000 Dijon, France

\begin{abstract}
Objective: In addition to its role in energy production and metabolism, mitochondria play a major role in apoptosis, oxidative stress, and calcium homeostasis. This review highlights the intricate role of mitochondria derived peptides (MPs), oxidative stress, and age-related disease such as diabetes.

Key Findings: The mitochondria produce MDPs: specific peptides that mediate transcriptional stress response by its translocation into the nucleus and interaction with DNA. MDPs are regulators of metabolism with cytoprotective effects through anti-oxidative stress, anti-inflammatory responses and anti-apoptosis. This class of peptides comprises: humanin (HN), MOTS-c, Small HN-like peptides. HN inhibits mitochondrial complex 1 activity and limits oxidative stress level in the cell. HN has been shown to prevent apoptosis by decreasing the reactive oxygen species production. Mitochondrial dysfunction and oxidative stress are implicated in the pathogenesis of diabetes. Data suggested that MDPs had a role in improving type 2 diabetes (T2D).
\end{abstract}

Summary: The goal of this review is to discuss the newly emerging functions of MDPs and their biological role in ageing and age-related diseases such as T2D.

Keywords: Mitochondrial-Derived-Peptides; Humanin; Oxidative Stress; Diabetes

\section{Introduction}

Mitochondria play a critical role in maintaining cellular function by ATP production. In addition to its role in energy production and metabolism, mitochondria play a major role in apoptosis, oxidative stress, and calcium homeostasis. A mitochondrial stress signal, or a 'mitokine', could confer protection and promote survival, while priming the cell's readiness for subsequent insults with increasing severity. The term 'mitohormesis' for such a phenomenon has been created [1]. The mitochondrial unfolded protein response (UPRmt) is a central part of the "mitohormetic" response. The UPRmt may be an alternative way in relationship with mitochondria signal in the cell. The mitochondria produce some specific peptides that mediate transcriptional stress response by the translocation into the nucleus and interaction with DNA. Mitochondrial derived peptides (MDPs) are regulators of metabolism and various studies have shown that MDPs exerted cytoprotective effects through anti-oxidative stress, anti-inflammatory responses and anti-apoptosis [2,3]. The goal of this review is to discuss the newly emerging functions of MDPs and their biological role in ageing and metabolic diseases such as T2D.

\section{Mitochondrial Metabolism Modulation}

\section{Functions in the Mitonuclear Communication Pathways}

Mitochondria booked a portion of the original bacterial genomes that co-evolved with nuclear genome. However, mitochondria import over a thousand proteins encoded in the nuclear genome to maintain their diverse functions, reflecting their adjacent relationship [4]. 
The mitochondrial genome inherits bacterial-like traits: the DNA molecules (mtDNA) are circular, double stranded, small $(16,569$ nucleotides in humans) and compact. mtDNA contains 37 genes, including 22 tRNAs, 2 rRNAs (12S and 16S rRNA) and 13 mRNAs encoding the proteins of the electron transport chain [5]. The mtDNA has no introns but a few non-coding nucleotides between adjacent genes and small open reading frames that encode functional MDPs. This class of peptides comprises humanin (HN) and mitochondrial open reading frame of the $12 \mathrm{~S}$ rRNA-c (MOTS-c) and expands the expression of mitochondrial proteome [6]. It has been established that mitochondria can export peptides and also import cytosolic peptides. It is the class of "cell-penetrating peptides" designed also as "mitochondrial cell-penetrating peptides" [7]. Many age-induced processes and degenerative diseases are related to mitochondrial dysfunction, further highlighting the critical importance of this organelle [8]. Complex human diseases, including diabetes, obesity, fatty liver disease and aging-related degenerative diseases are associated with alterations in mitochondrial oxidative phosphorylation (OXPHOS) function.

\section{Overview on Concepts of Retrograde Signaling and Unfolded Protein}

Numerous implications of these anterograde and retrograde signaling pathways between the mitochondria and the nucleus are appropriate for therapeutic exploitation with bioactive molecules.

\section{Concept of Retrograde Signaling}

The hallmark of mitochondrial retrograde signaling is the modification of the expression of nuclear genes induced by a signal from mitochondria [9]. Retrograde signaling must be triggered by a mitochondrial signal that in turn is relayed via molecules that finally reach the nucleus. In mammalian cells, altered nuclear expression in response to mitochondrial dysfunction is reported; a number of signaling pathways being implicated in this retrograde communication [10]. Mitochondrial retrograde signaling is a signaling pathway connecting mitochondria and the nucleus. Signal transducers in the yeast retrograde response are Rtg1p, Rtg2p, and Rtg3p proteins [11]. The outcomes of mitochondrial retrograde signaling go far beyond the maintenance or biogenesis of the organelle, affecting the homeostasis of the whole organism through body weight or immunity.

\section{Concept of Unfolded Protein}

Mitochondrial protein homeostasis is maintained through proper folding and assembly of newly translated polypeptides. Several factors challenge the mitochondrial protein-folding environment including reactive oxygen species (ROS) that are generated within mitochondria, as well as environmental situations such as exposure to toxic compounds. To promote efficient mitochondrial protein folding mitochondria possess molecular chaperones located in both the intermembrane space and matrix [12].
UPRmt is a mitochondria-to-nuclear communication mechanism that promotes adaptive regulation of nuclear genes related to mitochondrial response, and metabolism, implicated in the cellular homeostasis [13].

\section{Mitochondrial-Derived Peptides: Classification}

MDPs are a series of peptides encoded by mitochondrial DNA. This class of peptides comprises HN, MOTS-c, Small HN-like peptides (SHLPs) and expands the expression of mitochondrial proteome [6].

\section{Humanin}

The first MDP discovered back in 2001 was HN; the term based on the potential of this peptide for restoring the "humanity" of Alzheimer's disease (AD) patients. HN promotes cell survival in response to a variety of insults.

It is a small, secreted, 24 or 21 amino acid peptide, depending on cytoplasmic or mitochondrial translation, respectively. If HN is translated within the mitochondria, the peptide will be 21 amino acids; and if it is translated in the cytoplasm, then the result is a 24 amino acid peptide [14]. HN is encoded by an HN open reading frame (ORF) within the gene for the $16 \mathrm{~S}$ ribosomal subunit within the mitochondrial genome [15]. HN was discovered during a search for survival factors in unaffected areas of an AD patient's brain. The initial studies were first performed in cell culture and then followed by in vivo studies using both pharmacological mimetics of $\mathrm{AD}$ as well as mutant gene: amyloid- $\beta$ precursor protein. The most recent studies used transgenic models of AD. As HN is a relatively short peptide, exhaustive mutational analysis of the importance of each amino acid has been possible. Interestingly, single amino acid substitutions of HN can lead to significant alterations in its potency and biologic functions. S14G-HN in which the serine at position 14 is replaced by glycine, is a highly potent analogue of $\mathrm{HN}$.

Finally, HN may be the first small peptide of its kind representing a putative set of MDPs, a novel concept that modifies the established concept about retrograde mitochondrial signaling as well as mitochondrial gene expression. HN is a neuroprotective peptide and a cytoprotective factor against oxidative stress [16].

\section{Mitochondrial Open Reading Frame of the 12S rRNA-c ( MOTS-c)}

In addition to $\mathrm{HN}$, an in-silico search of the mitochondrial genome revealed additional potential MDPs. MOTS-c is expressed in various tissues and in circulation (plasma) in rodents and humans, suggesting both a cell-autonomous and hormonal role. Its primary target organ appears to be skeletal muscle and fat. The mitochondrially derived peptide MOTS-c was recently discovered. It is a 16 amino acid peptide located in the 12S rRNA gene. The first 11 amino acid residues of MOTS-c are highly conserved in 14 mammalian species [17]. MOTS-c has been identified as a 
gene expression regulator in the nucleus, leading to retrograde signaling via its interaction with transcription factors. MOTS-c polymorphism has been found to be associated with human longevity [18]. MOTS-c can prevent insulin resistance, dietmediated obesity, and ameliorate diabetes. MOTS-c oxidizes fatty acids and inhibits oxidative respiration [19]. MOTS-c increased the levels of carnitine metabolism, which transport activated fatty acids into the mitochondria for $\beta$-oxidation, increased the level of a $\beta$-oxidation intermediate. MOTS-c inhibited the folate cycle at the level of 5Me-THF, resulting in an accumulation of 5-aminoimidazole4-carboxamide ribonucleotide, an AMP-activated protein kinase (AMPK) activator. MOTS-c also increased cellular $\mathrm{NAD}^{+}$levels, which are nucleotide precursors $[17,20]$. MOTS-c regulated a broad range of genes in response to glucose restriction, including those with antioxidant response elements (ARE), and interacted with ARE-regulating stress-responsive transcription factors [21].

\section{Small HN-like Peptides}

Recently, six additional peptides encoded within the mitochondrial 16S rRNA region of mtDNA have been discovered and designed as SHLP1-6. SHLP2 and SHLP3 share similar biological effects with HN. The circulating levels of MOTS-c and SHLP2 decline with age. Various studies suggest that SHLP2 and SHLP3 may participate in the pathogenesis of age-related neurodegenerative diseases. The anti-oxidative stress function of SHLP2, and its neuroprotective effect indicate that SHLP2 has a role in the regulation of aging and age-related diseases [5].

\section{Ageing and Plasma MDPs Levels}

Ageing and longevity are or are not characterized by high levels of MDPs? It is speculated that MDPs production turns from protective to detrimental adaptive response; in these conditions, the levels increasing during aging. In some studies, HN levels significantly decline with age in humans. Plasma HN level was significantly lower in the older group $(1.3 \pm 0.2 \mathrm{ng} / \mathrm{mL})$ than that of the younger group $(1.7 \pm 0.1 \mathrm{ng} / \mathrm{mL})$ [22]. In other studies, it is reported that HN levels significantly decline with age in humans and animals. HN levels in plasma were measured in young and old mice and across age in humans. HN levels decreased with age in both mice and human (Human plasma levels: 45-65 years: 1400 pg/mL; 80-110 years: $1000 \mathrm{pg} / \mathrm{mL}$ ) [5].

New results are in contrast with these data. HN plasma levels are evaluated in 693 subjects aged from 21 to 113 years. HN levels increased in old age $(>500 \mathrm{pg} / \mathrm{mL})$, with the highest levels found in centenarians (> $1000 \mathrm{pg} / \mathrm{mL}$ ). The plasmatic levels of $\mathrm{HN}$ are significantly positively correlated with age. No gender differences were observed for HN. HN plasma level is associated negatively with body mass index in elderly patients [23]. Concerning the other MDPs, it is reported that MOTS-c and SHLP2 circulating levels decline with age. The circulating SHLP2 levels significantly decreased with age in both male and female C57BL/ 6 mice (young, 3 months old: 3000 pg/mL; aged, 18 months old: $2500 \mathrm{pg} / \mathrm{mL}$ ). Male mice had higher SHLP2 levels than female mice in both the young and old groups [5]. The results of these studies should be interpreted considering the following limitations. First, the relatively small sample size in each group represents a potential limitation. Second, mitochondrial diseases are an expanding group of disorders with many metabolic deficiencies. In the ideal case, the used patient cohort should display a homogeneous phenotype, disease stage, and organ specificity. Moreover, the discovery of ageing-related biomarkers is supported by the development of advanced proteomics technology. Changes in the circulating concentrations of human proteins can serve as predictive measures of health and disease [24].

\section{Mechanisms of Action of MDPs}

MDPs exert functions through binding to both intracellular molecules and putative cell membrane receptors.

\section{MDPs Receptors}

Emerging studies show that MDPs play important roles in cytoprotection and homeostasis. HN has been shown to increase extracellular signal-regulated kinase 1/2 (ERK1/2) phosphorylation through its receptor binding [25]. The ERK1/2 cascade serves as an essential mediator in a lot of cellular processes such as proliferation, cell migration, cellular metabolism, and survival. Upon stimulation, ERK1/2 is phosphorylated and becomes dissociated from its anchoring proteins, allowing the translocation of ERK1/2 to other subcellular compartments. HN has been shown to act as a ligand to two different types of receptors; the seven-transmembrane G-protein-coupled receptor formyl-peptide receptor-like-1 (FPRL1), and a trimeric receptor consisting of ciliary neurotrophic factor receptor (CNTFR), the cytokine receptor WSX-1 and the transmembrane glycoprotein gp130 (CNTFR/WSX1/gp130) [26,27].

The first HN receptor FPRL1 has been linked to AD. HN acts as an agonist for FPRL1 by inducing $\mathrm{Ca}^{2+}$ mobilization and activation of ERK1/2 signaling, the pathway of G-protein coupled receptors, which participate to its cytoprotective properties [26]. The second reported HN receptor is the trimeric CNTFR/WSX-1/gp130 complex. The activation of the gp130-STAT3 axis is essential for HN activity. HN induces STAT3 activation, which was required for its neuroprotective effects [27]. Gp130 is part of the receptor complex for several cytokines, including IL-6, IL-27.

Concerning the cytoprotective effects of HN or S14G-HN (HN derived), studies suggest that this protection may be mediated through activation of AMPK in thrombin-mediated activation of endothelial nitric-oxide synthase (eNOS) signaling as well as reduction of pro-apoptotic factors [28]. HN in actives proapoptotic peptides such as Bax. It prevents Bax translocation and activation in response to proapoptotic agents [29]. 


\section{Signaling Pathways and Interface with IGF-I}

$\mathrm{HN}$ is regulated by insulin-like growth factor-I (IGF-I) and growth hormone (GH). HN and IGF-I levels simultaneously decrease with age and it has been demonstrated that HN levels are directly down-regulated by IGF-I [30]. The biological activity of IGF-I is controlled by six binding proteins: insulin-like growth factorbinding protein (IGFBPs), preventing the IGFs from interacting with the IGF receptor. IGF-binding proteins are a group of proteins that regulates IGF-I bioavailability by acting as carrier, in particular IGFBP-3. HN binds to IGFBP-3 with high affinity and specificity. HN protects against IGFBP-3 induced apoptosis, recent studies suggesting a possible mechanism of action for $\mathrm{HN}$ as an inhibitor of IGFBP3 nuclear translocation [31].

Finally, intracellularly MDPs interact with proteins such as Bax as related previously but also to bind IGFBP-3. Thereby inhibiting IGFBP-3 dependent cell death [31]. Concerning the relationship between MDPs and GH metabolism; it has been suggested that GH inhibits levels via IGF-I. Recent review described the role of nutrition and insulin in determining the regulation and actions of the GH-IGF-axis [32]. Excess insulin, GH, IGF-I, and IGF-II signaling are associated with severe metabolic disease and excess growth and/or obesity. Situation such as malnutrition is associated with a decrease insulin signaling which reduces growth hormone receptor expression blocking the GH signaling pathway. Overproduction of GH has been linked to cancer and the microvascular complications that are associated with diabetes [33].

\section{MDPs Activity, Oxidative Stress and Type 2 Diabetes}

Various studies have shown that MDPs exert cytoprotective effects through anti-oxidative stress, anti-inflammatory responses and anti-apoptosis. Oxidative stress has been implicated in a multitude of diseases, and because of the success of HN treatment against amyloid-beta stress, several studies have tested the relationship between HN activity and oxidative stress.

It is commonly accepted that the majority of cellular ROS originates from mitochondrial energy metabolism. Partial reduction of $\mathrm{O}_{2}$ yields the superoxide anion; it is estimated that $\sim 0.2-2 \%$ of $\mathrm{O}_{2}$ consumed by mitochondria is converted to superoxide. Bioenergetics' function of mitochondria is associated with generation of adenosine triphosphate (ATP) via OXPHOS system. Electrons are transferred from NADH, produced in the citric acid cycle in the mitochondrial matrix, to $\mathrm{O}_{2}$ by a series of large protein complexes in the inner mitochondrial membrane. The two major sites for electron leakage are OXPHOS complexes I and III. Generation of ATP through mitochondrial oxidative phosphorylation is central to the maintenance of the optimal ATP/ adenosine diphosphate ratios within the cell. Oxidative stress results from either an increase in free radical production, or a decrease in endogenous antioxidant defenses, or both. ROS and reactive nitrogen species (RNS) are products of cellular metabolism and are well recognized for their dual role as both deleterious and beneficial species.

There is evidence that ROS/RNS play key roles in the pathogenesis of various diseases. Oxidative stress by definition is due to an overproduction of ROS/RNS and/or a deficiency in enzymatic and non-enzymatic antioxidant defense, such that the balance is rolled in favor of ROS/RNS accumulation and cell damage $[34,35]$.

Oxidative stress is known to contribute to a range of harmful intracellular events, including DNA damage within the nucleus. This damage may ultimately cause organ dysfunction, and cell death. Enhanced generation of ROS and oxidative stress occurs in mitochondria as a consequence of an overload of glucose and oxidative phosphorylation. Endoplasmic reticulum (ER) stress plays an important role in oxidative stress, as it is also a source of ROS. The tight interconnection between mitochondria and ER means that the ROS generated in mitochondria promote ER stress. The recent discovery of interconnections between the ER membrane and those of almost all the cell compartments is providing novel perspectives for the understanding of the molecular events in both physiological and pathological conditions [36,37]. ER stress and oxidative stress are participants in an array of physiological and pathophysiological conditions. The retinal pigment epithelium (RPE) is implicated in the age-related macular degeneration relationship with increase of oxidative stress levels. ER stress and oxidative stress induce RPE apoptosis by increased generation of ROS and activation of caspase 3. HN treatment inhibits caspases activities and upregulates glutathione (GSH) [38]. GSH is a tripeptide, which has many biological roles including a major protection against ROS and RNS [39]. The reduced and oxidized forms of glutathione (GSH and GSSG) act in concert with other redox-active compounds (NAD(P) H) to regulate and maintain cellular redox status.

Recently it has been demonstrated that HNG: an HN analogue was able to prevent against mitochondrial dysfunction in conditions of oxidative stress in a cell-free system. The application of HNG in isolated cardiac mitochondria led to attenuated mitochondrial dysfunction against $\mathrm{H}_{2} \mathrm{O}_{2}$ induced oxidative stress.

In these conditions the protective actions are indicated by

1) decreased mitochondrial ROS level;

2) attenuated mitochondrial depolarization;

3) reduced mitochondrial swelling; and

4) increased mitochondrial ATP production [40].

The properties of MDPs on oxidative stress are supported by findings in cellular and rodent models: $\mathrm{HN}$ inhibiting oxidative stress, rescues mitochondrial function, and lowers apoptotic rate. It has been shown that HNG enhances intracellular antioxidant capacity, preserves mitochondrial membrane potential, ATP levels 
and restores mitochondrial integrity in rat's myoblasts (H9c2 cells) [41]. HNG inhibits mitochondrial complex 1 activity and prevents mitochondrial dysfunction and oxidative stress induced by $\mathrm{H}_{2} \mathrm{O}_{2}$ in isolated cardiac mitochondria [40].

Mitochondrial dysfunction and oxidative stress are implicated in the pathogenesis of diabetes [35,42]. The exact mechanisms underlying the disease are unknown; however, there is growing evidence that excess generation of ROS, largely due to hyperglycemia, causes oxidative stress in a variety of tissues. In type 2 diabetic (T2D) patients, oxidative stress is closely associated with chronic inflammation. Multiple signaling pathways contribute to the adverse effects of glucotoxicity on cellular functions.

Data suggested that MDPs had a role in improving T2D [17]. The aim of recent studies was to determine MDP levels in normal, prediabetes and diabetes subjects. The MDPs: HN and MOT-c, similar to ADP, are decreased in T2D and correlate with HbA1c. The data provide an additional evidence that mitochondrial dysfunction contributes to glycemic dysregulation and metabolic defects in T2D [43]. The lower levels of $\mathrm{HN}$ in T2D subjects might be a secondary response to the elevated ROS production, and oxidative stress. Altered HN levels in T2D could serve as a potential biomarker.

Administration of HN and a HN analogue (HNG-F6A) reduced blood glucose and promoted insulin sensitivity in diabetic rats. The favorable effects of MOTS-c on insulin sensitivity and glucose metabolism have been seen in animal models, with MOTS-c enhancing glucose utilization, promotion of insulin sensitivity and restoration of metabolic homeostasis [17]. It is possible that marked increase in ROS levels may act to recruit HN from several tissues in the body to damaged areas. In these conditions, HN exerts an antioxidative stress action; this effect could preserve cell survival. In human aortic endothelial cells, HN has been shown to prevent apoptosis by decreasing the ROS production [41].

\section{Role for MDPs in the Regulation of Inflammatory Responses}

In relationship with the oxidative stress, a role for MDPs in down-regulation of inflammatory responses has been demonstrated in vivo and in cell cultures. Inflammation is associated with overabundance of ROS in the cell, which can lead to damage of cellular components, and activation of cell death pathways. To elude the damaging effect of ROS, cells have developed several defense mechanisms with the scope of scavenging free radicals. To maintain ROS and RNS at a non-toxic concentration, enzymatic and nonenzymatic cellular antioxidants coordinate the balance between their production and their degradation. A variety of transcription factors may be activated depending on the level of oxidative stress, leading to the expression of different genes. Redox imbalance, caused by increased free radical's production and/or reduced antioxidant defense, plays an important role in the development of various diseases. The redox state of the cell is predominantly dependent on an iron redox couple and is maintained within strict physiological limits $[44,45]$.

MOTS-c inhibited pro-inflammatory cytokines such as IL-6 and IL-1 $\beta$ through decreasing the phosphorylation of mitogenactivated protein kinases (MAPK) [17]. The systemic effects of the administration of MOTS-c as a daily injection in aged mice suggest that this molecule might be useful in ameliorating the abnormal metabolism associated with aging [19]. Increased oxidative stress, one of the major factors contributing to cell death, plays an important role in the inflammatory process of atherosclerosis. In this field, a study showed that in a cell culture model using human amniotic epithelial cells, pre-treatment with $\mathrm{HN}$ attenuated OxLDL-induced ROS formation and apoptosis by 50\% [22]. Recently a study investigated HN and MOTS-c protein expression in skeletal muscle and serum levels in advanced chronic kidney disease (CKD) patients and age-matched controls with normal renal function. MOTS-c levels were reduced in both skeletal muscle and serum in CKD. In return, circulating levels of HN were increased in CKD and correlated positively to circulating inflammatory mediator such as tumor necrosis factor-alpha (TNF- $\alpha$ ) levels [46].

Upon exposure to high glucose, vascular endothelial cells (ECs) produce high levels of ROS and proinflammatory cytokines such as TNF- $\alpha$ and IL-1 $\beta$ [47]. ECs respond to high glucose stimuli by causing the expression of chemokines and adhesion molecules, which induce leukocyte attachment and rolling on the endothelial surface [48]. Experimental studies suggest that HN may have therapeutic potential for the treatment of hyperglycemia associated endothelial dysfunction according to their anti-inflammatory properties. HN treatment inhibited high glucose-induced secretion of TNF- $\alpha$ and IL-1 $\beta$. This reduction of the expression of these proinflammatory cytokines is associated with a reduction of the expression of vascular cell adhesion molecule 1 (VCAM-1) and E-selectin [49]. The repression of high glucose-induced monocyte adhesion by HN was mediated by Krüppel-like factor-2 (KLF2). KLF2 is a transcription factor that plays a major role in the regulation of EC function. KLF2 protects against EC injury through its anti-inflammatory effects to maintain the normal vascular integrity [50].

\section{Conclusions and Perspectives}

The discovery of MDPs as a novel, stress-responsive, and cytoprotective peptides derived from a mitochondrial ORF has opened up a new field of research. In the cardiovascular field, $\mathrm{HN}$ levels act to preserve coronary endothelial function and HN level may be used as a biomarker for the early development of atherosclerosis [22,51]. Data suggested that MDPs had a role in improving T2D. Studies provide an additional evidence that mitochondrial dysfunction contributes to glycemic dysregulation and metabolic defects in T2D. Concerning the brain area, according various reports, HN and its derivatives are peptides known for their protective antiapoptotic effects. These compounds are 
potential neuroprotective agents in treating $\mathrm{AD}$ and amyotrophic lateral sclerosis (ALS). However, in return; the studies of MDPs in cardiometabolic diseases and neurodegenerative disorders are still in the experimental stage.

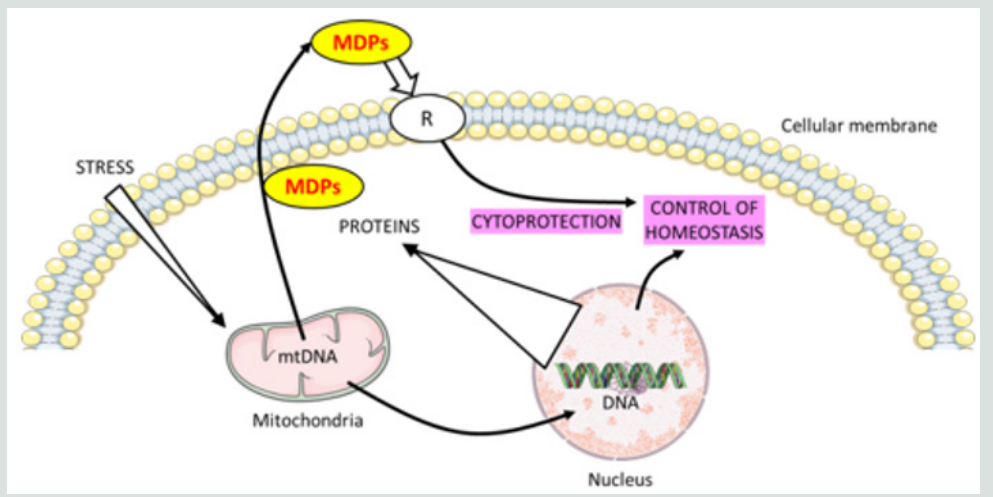

Figure 1: Intracellular and extracellular mechanisms of action of mitochondrial derived peptides (MDPs).

The mitochondrial genome is regulated by factors encoded in the nucleus. Mitochondria communicate with the cell through a process of retrograde signaling. MDPs are retrograde signaling molecules. They act as endocrine as well as intracellular factors and the function of these MDPs is to participate to homeostasis control via the activation of signaling pathways.

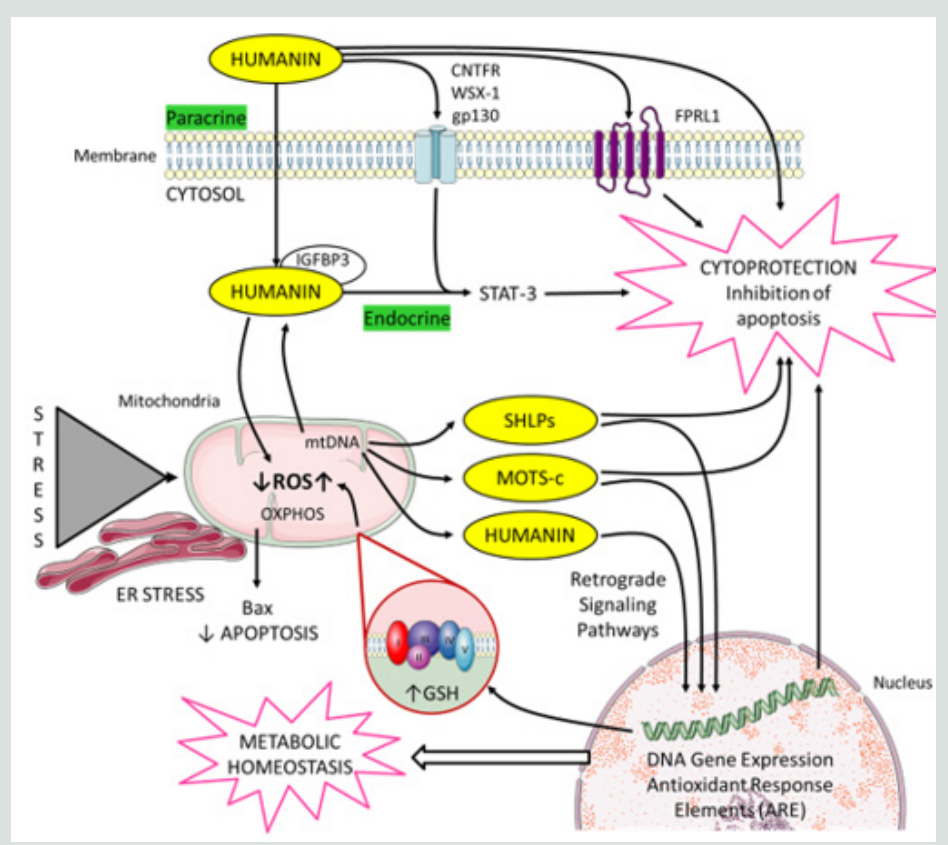

Figure 2: Cellular actions of mitochondrial derived peptides (MDPs).

MDPs meet the criteria of a "mitokine" because their biogenesis is linked to mitochondria, and they have a paracrine and endocrine impact on the whole organism. The figure illustrates how mitochondria communicate with the nucleus. Mitochondria also communicate with other organelles such as endoplasmic reticulum to coordinate cellular processes. This includes MDPs, and mitochondrial reactive oxygen species (ROS). MDPs include humanin (HN), MOTS-c (mitochondrial open reading frame of the $12 \mathrm{~S}$ rRNA-c), and Small HN-like peptides (SHLPs).

HN presents both intra-and extra-cellular modes of action. Within a cell, HN interacts with proapoptotic proteins such as Bax and IGFBP-3. Extracellular HN activates two types of cell-surface receptors; a trimeric receptor involving CNTFR/WSX-1/ gp130 and relays through the STAT3 signaling pathway. The second HN receptor: FPRL1 has been linked to Alzheimer's disease. HN directly binds to amyloid-beta 17-28 (A beta 17-28).

Generation of mitochondrial ROS takes place at the electron transport chain located on the inner mitochondrial membrane during the process of oxidative phosphorylation (OXPHOS). Respiratory chain complexes I and III are the main producers of ROS. MDPs regulated some genes in response to oxidative stress via antioxidant response elements (ARE). HN regulates cellular oxidative stress; decreases mitochondrial ROS and increases mitochondrial GSH. 


\section{Declaration of Competing Interest}

The authors declare no conflict of interest.

This work was supported by grants from French Ministry of Research, and from the Conseil Régional de Bourgogne-FrancheComté, FEDER, Dijon University Hospital Center and Association de Cardiologie de Bourgogne. The authors wish to thank Suzanne Rankin for English assistance.

\section{References}

1. Ristow M, Zarse K (2010) How increased oxidative stress promotes longevity and metabolic health: The concept of mitochondrial hormesis (mitohormesis). Exp Gerontol 45(6): 410-418.

2. Yong C Q Y, Tang B L (2018) A Mitochondrial Encoded Messenger at the Nucleus. Cells 7(8): 105.

3. Kim S J, Xiao J, Wan J, Cohen P, Yen K (2017) Mitochondrially derived peptides as novel regulators of metabolism. J Physiol 595(21): 66136621

4. Son J M, Lee C (2019) Mitochondria: multifaceted regulators of aging BMB reports 52(1): 13-23.

5. Cobb L J, Lee C, Xiao J, Yen K, Wong R G, et al. (2016) Naturally occurring mitochondrial-derived peptides are age-dependent regulators of apoptosis, insulin sensitivity, and inflammatory markers. Aging (Albany NY) 8(4): 796-809.

6. Kim S J, Mehta H H, Wan J, Kuehnemann C, Chen J, et al. (2018) Mitochondrial peptides modulate mitochondrial function during cellular senescence. Aging (Albany NY) 10(6): 1239-1256.

7. Jain A, Chugh A (2016) Mitochondrial transit peptide exhibits cell penetration ability and efficiently delivers macromolecules to mitochondria. FEBS Lett 590(17): 2896-2905.

8. Kamat P K, Kalani A, Kyles P, Tyagi S C, Tyagi N (2014) Autophagy of mitochondria: a promising therapeutic target for neurodegenerative disease. Cell Biochem Biophys 70(2): 707-719.

9. Da Cunha F M, Torelli N Q, Kowaltowski A J (2015) Mitochondrial Retrograde Signaling: Triggers, Pathways, and Outcomes. Oxidative medicine and cellular longevity 482582.

10. Butow R A, Avadhani N G (2004) Mitochondrial signaling: the retrograde response. Mol Cell 14(1): 1-15.

11. Trendeleva T A, Zvyagilskaya R A (2018) Retrograde Signaling as a Mechanism of Yeast Adaptation to Unfavorable Factors. Biochemistry (Mosc) 83(2): 98-106.

12. Fujimoto M, Nakai A (2010) The heat shock factor family and adaptation to proteotoxic stress. The FEBS journal 277(20): 4112-4125.

13. Yi H S, Chang J Y, Shong M (2018) The mitochondrial unfolded protein response and mitohormesis: a perspective on metabolic diseases. J Mol Endocrinol 61(3): R91-R105.

14. Yamagishi Y, Hashimoto Y, Niikura T, Nishimoto I (2003) Identification of essential amino acids in Humanin, a neuroprotective factor against Alzheimer's disease-relevant insults. Peptides 24(4): 585-595.

15. Bodzioch M, Lapicka-Bodzioch K, Zapala B, Kamysz W, Kiec-Wilk B, et al. (2009) Evidence for potential functionality of nuclearly-encoded humanin isoforms. Genomics 94(4): 247-256.

16. Lee C, Yen K, Cohen P (2013) Humanin: a harbinger of mitochondrialderived peptides? Trends Endocrinol Metab 24(5): 222-228.

17. Lee C, Zeng J, Drew B G, Sallam T, Martin-Montalvo A, et al. (2015) The mitochondrial-derived peptide MOTS-c promotes metabolic homeostasis and reduces obesity and insulin resistance. Cell Metab 21(3): 443-454.

18. Fuku N, Pareja-Galeano H, Zempo H, Alis R, Arai Y, et al. (2015) The mitochondrial-derived peptide MOTS-c: a player in exceptional longevity? Aging Cell 14(6): 921-923

19. Yang Y, Gao H, Zhou H, Liu Q, Qi Z, et al. (2019) The role of mitochondriaderived peptides in cardiovascular disease: Recent updates. Biomedicine \& pharmacotherapy 117: 109075.

20. Lee C, Kim K H, Cohen P (2016) MOTS-c: A novel mitochondrial-derived peptide regulating muscle and fat metabolism. Free Radic Biol Med 100: 182-187.

21. Kim K H, Son J M, Benayoun B A, Lee C (2018) The MitochondrialEncoded Peptide MOTS-c Translocates to the Nucleus to Regulate Nuclear Gene Expression in Response to Metabolic Stress. Cell Metab 28(3): 516-524.e7.

22. Bachar A R, Scheffer L, Schroeder A S, Nakamura H K, Cobb L J, et al. (2010) Humanin is expressed in human vascular walls and has a cytoprotective effect against oxidized LDL-induced oxidative stress. Cardiovasc Res 88(2): 360-366.

23. Conte M, Ostan R, Fabbri C, Santoro A, Guidarelli G, et al. (2019) Human Aging and Longevity Are Characterized by High Levels of Mitokines. J Gerontol A Biol Sci Med Sci 74(5): 600-607.

24. Byerley L O, Leamy L, Tam S W, Chou C W, Ravussin E, et al. (2010) Development of a serum profile for healthy aging. Age (Dordr) 32(4): 497-507.

25. Kim S J, Guerrero N, Wassef G, Xiao J, Mehta H H, et al. (2016) The mitochondrial-derived peptide humanin activates the ERK1/2, AKT, and STAT3 signaling pathways and has age-dependent signaling differences in the hippocampus. Oncotarget 7(30): 46899-46912.

26. Ying G, Iribarren P, Zhou Y, Gong W, Zhang N, et al. (2004) Humanin, a newly identified neuroprotective factor, uses the $G$ protein-coupled formylpeptide receptor-like-1 as a functional receptor. J Immunol 172(11): 7078-7085

27. Hashimoto Y, Kurita M, Aiso S, Nishimoto I, Matsuoka M (2009) Humanin inhibits neuronal cell death by interacting with a cytokine receptor complex or complexes involving CNTF receptor alpha/WSX-1/gp130. Mol Biol Cell 20(12): 2864-2873.

28. Muzumdar R H, Huffman D M, Calvert J W, Jha S, Weinberg Y, et al. (2010) Acute humanin therapy attenuates myocardial ischemia and reperfusion injury in mice. Arterioscler Thromb Vasc Biol 30(10): 1940-1948.

29. Guo B, Zhai D, Cabezas E, Welsh K, Nouraini S, et al. (2003) Humanin peptide suppresses apoptosis by interfering with Bax activation. Nature 423(6938): 456-461.

30. Xiao J, Kim S J, Cohen P, Yen K (2016) Humanin: Functional Interfaces with IGF-I. Growth Horm IGF Res 29: 21-27.

31. Njomen E, Evans H G, Gedara S H, Heyl D L (2015) Humanin Peptide Binds to Insulin-Like Growth Factor-Binding Protein 3 (IGFBP3) and Regulates Its Interaction with Importin-beta. Protein Pept Lett 22(10): 869-876.

32. Clayton P E, Banerjee I, Murray P G, Renehan A G (2017) Growth hormone, the insulin-like growth factor axis, insulin and cancer risk. Nat Rev Endocrinol 7(1): 11-24.

33. Lu M, Flanagan J U, Langley R J, Hay M P, Perry J K (2019) Targeting growth hormone function: strategies and therapeutic applications. Signal Transduct Target Ther 4: 3 .

34. Rochette L, Lorin J, Zeller M, Guilland J C, Lorgis L, et al. (2013) Nitric oxide synthase inhibition and oxidative stress in cardiovascular diseases: possible therapeutic targets? Pharmacol Ther 140 (3): 239-257. 
35. Rochette L, Zeller M, Cottin Y, Vergely C (2018) Redox Functions of Heme Oxygenase-1 and Biliverdin Reductase in Diabetes. Trends Endocrinol Metab 29(2): 74-85.

36. Burgos-Moron E, Abad-Jimenez Z, Maranon A M, Iannantuoni F, Escribano-Lopez I, et al. (2019) Relationship Between Oxidative Stress, ER Stress, and Inflammation in Type 2 Diabetes: The Battle Continues. J Clin Med 8(9): 1385.

37. Moltedo O, Remondelli P, Amodio G (2019) The MitochondriaEndoplasmic Reticulum Contacts and Their Critical Role in Aging and Age-Associated Diseases. Front Cell Dev Biol 7: 172.

38. Minasyan L, Sreekumar P G, Hinton D R, Kannan R (2017) Protective Mechanisms of the Mitochondrial-Derived Peptide Humanin in Oxidative and Endoplasmic Reticulum Stress in RPE Cells. Oxidative medicine and cellular longevity 1675230.

39. Rochette L, Vergely C (2016) Coronary artery disease: Can aminothiols be distinguished from reactive oxygen species? Nature reviews. Cardiology 13(3): 128-130.

40. Thummasorn S, Shinlapawittayatorn K, Khamseekaew J, Jaiwongkam T, Chattipakorn S C, et al. (2018) Humanin directly protects cardiac mitochondria against dysfunction initiated by oxidative stress by decreasing complex I activity. Mitochondrion 38: 31-40.

41. Klein L E, Cui L, Gong Z, Su K, Muzumdar R (2013) A humanin analog decreases oxidative stress and preserves mitochondrial integrity in cardiac myoblasts. Biochem Biophys Res Commun 440(2): 197-203.

42. Rochette L, Zeller M, Cottin Y, Vergely C (2014) Diabetes, oxidative stress and therapeutic strategies. Biochimica et biophysica acta 1840(9): 2709-2729.

43. Ramanjaneya M, Bettahi I, Jerobin J, Chandra P, Abi Khalil C, et al. (2019) Mitochondrial-Derived Peptides Are Down Regulated in Diabetes Subjects. Front Endocrinol (Lausanne) 10: 331.

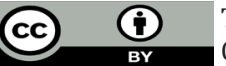

This work is licensed under Creative Commons Attribution 4.0 License

To Submit Your Article Click Here:

Submit Article
DOI: $10.32474 / \mathrm{AD} 0.2020 .03 .000155$
44. Gudjoncik A, Guenancia C, Zeller M, Cottin Y, Vergely C, et al. (2014) Iron, oxidative stress, and redox signaling in the cardiovascular system. Molecular nutrition \& food research 58(8): 1721-1738.

45. Rochette L, Gudjoncik A, Guenancia C, Zeller M, Cottin Y, et al. (2015) The iron-regulatory hormone hepcidin: a possible therapeutic target? Pharmacol Ther 146: 35-52.

46. Liu C, Gidlund E K, Witasp A, Qureshi A R, Soderberg M, et al. (2019) Reduced skeletal muscle expression of mitochondrial-derived peptides humanin and MOTS-C and Nrf2 in chronic kidney disease. Am J Physiol Renal Physiol 317(5): F1122-F1131.

47. Lenna S, Han R, Trojanowska M (2014) Endoplasmic reticulum stress and endothelial dysfunction. IUBMB life 66(8): 530-537.

48. Kang H, Ma X, Liu J, Fan Y, Deng X (2017) High glucose-induced endothelial progenitor cell dysfunction. Diabetes \& vascular disease research : official journal of the International Society of Diabetes and Vascular Disease 14(5): 381-394.

49. Wang X, Wu Z, He Y, Zhang H, Tian L, et al. (2018) Humanin prevents high glucose-induced monocyte adhesion to endothelial cells by targeting KLF2. Mol Immunol 101: 245-250.

50. Rane M J, Zhao Y, Cai L (2019) Krupsilonppel-like factors (KLFs) in renal physiology and disease. EBioMedicine 40: 743-750.

51. Yun K Oh, Bachar A R, Zacharias D G, Kim S G, Wan J, et al. (2011) Humanin preserves endothelial function and prevents atherosclerotic plaque progression in hypercholesterolemic ApoE deficient mice. Atherosclerosis 219(1): 65-73.

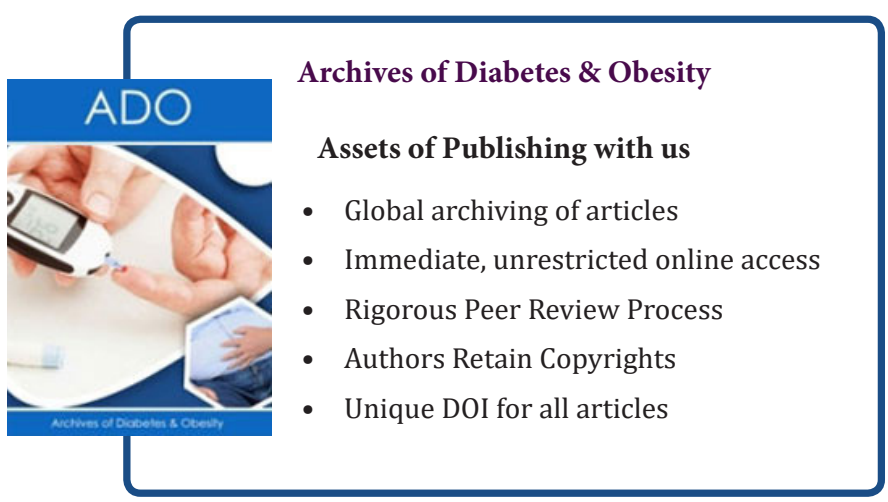

